\title{
L'idrogeologia italiana in movimento: Flowpath 2012
}

\author{
Marco Petitta
}

Presidente del Comitato Italiano IAH

Marco.Petitta@uniroma1.it

All'inizio dell'estate a Bologna si è riunita la comunità idrogeologica italiana, in occasione del Convegno Flowpath - Percorsi di Idrogeologia, organizzato dal prof.Alessandro Gargini del Dipartimento di Scienze della Terra e GeologicoAmbientali dell'Università di Bologna "Alma Mater Studiorum”, in collaborazione con la Regione Emilia Romagna, Servizio Geologico Sismico e dei Suoli, sotto l'egida del Comitato Italiano IAH. Era da diversi anni (probabilmente dagli storici congressi organizzati dal GNDCI sempre in Emilia Romagna negli anni '90) che mancava in Italia un evento specificamente dedicato all'idrogeologia, diretto sia alla comunità scientifica che ai professionisti ed operatori del settore. Dal numero dei partecipanti (ben 189 iscritti) si può affermare che se ne sentiva la mancanza.

Crediamo che i contenuti delle tre giornate del convegno abbiano ampiamente ripagato tutti di questa lunga attesa, offrendo un panorama aggiornato e ad ampio spettro delle attività idrogeologiche in campo scientifico, tecnico e professionale. La presenza di moltissimi giovani (precari della ricerca e professionisti), pari al 50\% degli iscritti, e la scelta organizzativa di far presentare i risultati degli studi esclusivamente ai giovani ricercatori, ha decisamente indirizzato il convegno verso un'atmosfera rilassata ma frizzante, non usuale negli ambienti accademici nostrani, e questo ha probabilmente giovato sia alla riuscita della manifestazione che soprattutto a rendere "fertile" la discussione, i confronti e le presentazioni. Proprio questo era lo scopo dichiarato dell'organizzazione, nel tentativo di rendere interattivo il contatto tra il mondo della ricerca e quello della professione, che nel settore delle acque sotterranee devono essere strettamente interconnessi.

Ad impreziosire il programma ci hanno pensato gli ospiti stranieri, tutti di indubbia esperienza e fama internazionale, che hanno presentato delle relazioni ad invito su temi di ampio respiro e proiettati verso il futuro dell'idrogeologia. Ramon Aravena dell'Università di Waterloo, Canada, ha descritto le potenzialità e le applicazioni degli isotopi ambientali in campo idrogeologico, con particolare riferimento alle problematiche di inquinamento e natural attenuation; Nico Goldscheider del Karlsruhe Institute of Technology, Germania, ha esposto il tema del monitoraggio e della caratterizzazione negli acquiferi carsici, mentre Beth Parker dell'Università di Guelph, Canada, ha trattato la problematica della contaminazione in acquiferi sedimentari fratturati. La sessione finale è stata affidata a John Cherry (Fig. 1), autore del famoso testo di idrogeologia "Groundwater" (Freeze \& Cherry, 1979) che ha relazionato sul ruolo degli aquitard nei sistemi idrogeologici complessi. Tutti gli interventi hanno suscitato le positive reazioni dei presenti, che hanno avuto l'occasione di rivolgere domande ai relatori, animando la discussione.

Le tre sessioni, comprensive delle esposizioni dei poster, hanno raccolto 85 contributi, che $\mathrm{i}$ giovani relatori hanno esposto e discusso di fronte ad un'ampia platea. I temi affrontati hanno riguardato la parametrizzazione degli acquiferi (tramite acquisizione di dati relativi alle caratteristiche

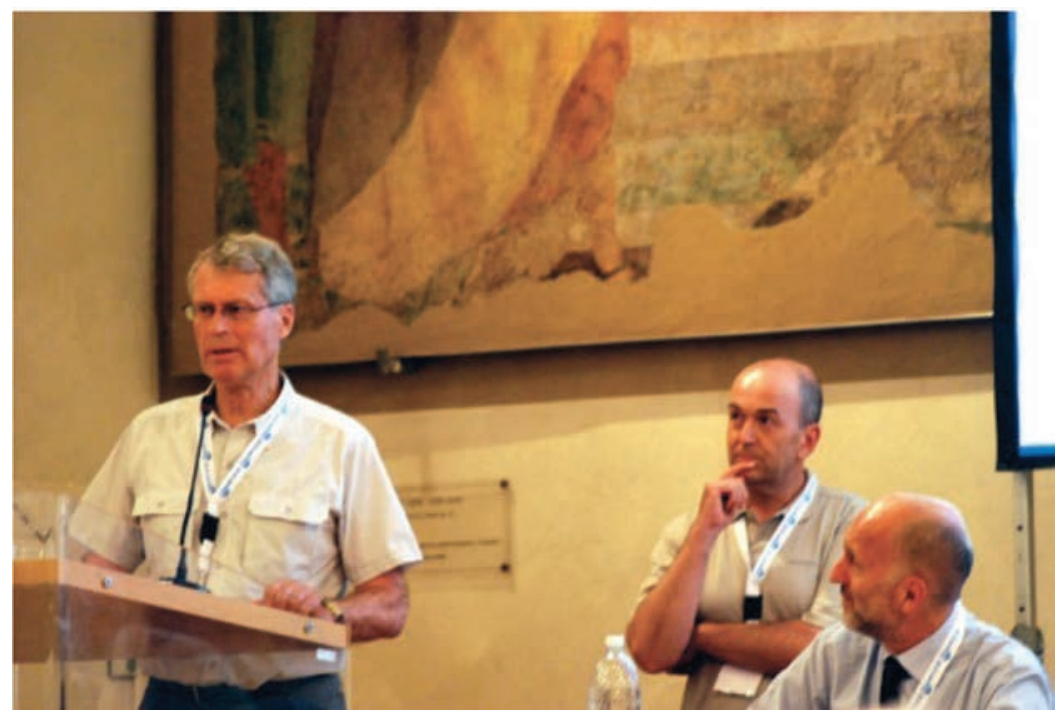

Fig. 1 - Un momento dell'intervento di John Cherry. 
idrogeologiche, ma anche organizzazione in database e GIS), il trasporto dei contaminanti nelle acque sotterranee (con casi di studio a scala di dettaglio e regionale, approcci modellistici ed esperimenti di laboratorio) e la modellistica idrogeologica e idrochimica (con applicazioni a casi reali, approcci statistici, ma anche modellazione delle componenti disciolte). I contributi presentati, oltre che essere presenti in forma di riassunto sugli atti del convegno, disponibili in rete sul sito della IAH Italiana (www.iahitaly.it), saranno raccolti e stampati in forma estesa nei prossimi numeri di questa rivista e di AQUAmundi - journal of water sciences, entrambe edite dall'Associazione Acque Sotterranee. Inevitabilmente, durante il convegno, un intervento speciale è stato dedicato, attraverso l'esposizione del Dott. Giovanni Bertolini del Servizio Geologico Regionale, alle prime riflessioni sugli aspetti idrogeologici connessi al terremoto del maggio scorso, che ha colpito l'Emilia-Romagna.

Durante il convegno i giovani ricercatori hanno avuto ulteriori occasioni di farsi notare con la consegna del Premio per Tesi di Dottorato intitolato a Gian Maria Zuppi, fortemente voluto dalla IAH Italiana e dall'IGAG-CNR, che è andato a Nicolò Colombani, de La Sapienza di Roma, attualmente assegnista di ricerca presso l'Università di Ferrara, oltre al quale si sono distinte anche le tesi di Valentina Carucci (La Sapienza di Roma) e di Viviana Re (Università Cà Foscari di Venezia). La consegna del premio è stata anche l'occasione per un commosso ricordo della figura di ricercatore di Gian Maria Zuppi, scomparso nel 2011. Un altro premio per il miglior poster è invece stato direttamente assegnato dalla giuria composta dagli ospiti internazionali ex-equo ad Emma Petrella dell'Università del Molise e a Cinzia Banzato \& Federico Marchionatti del Politecnico di Torino.

A cornice del programma scientifico, gli eventi sociali hanno contribuito a creare il clima di distensione e familiarità necessario per la buona riuscita del convegno, a partire dai coffee break e lunch direttamente organizzati nella prestigiosa sede dello splendido Complesso Monumentale di San Giovanni in Monte, per proseguire con le due cene, la prima nella cornice del Museo Geologico Giovanni Capellini, presso il Dipartimento di Scienze della Terra e Geologico-Ambientali, e la seconda nello storico ristorante Diana di Bologna. A conclusione del programma scientifico, il terzo giorno è stato dedicato ad un'escursione alle sorgenti dei colli bolognesi, completata da una visita a Porretta Terme nel pomeriggio. Il tutto sempre a cura dell'infaticabile staff coordinato da Alessandro Gargini e con la collaborazione della Regione Emilia-Romagna. Un ampio resoconto fotografico della manifestazione è disponibile sul sito della IAH Italiana (www.iahitaly.it)

Poche righe finali sul bilancio della manifestazione: a chi ha partecipato è sembrata ben riuscita, soprattutto se si considerano le prospettive che sono emerse dalla ampia partecipazione (Fig. 2), e dall'atmosfera fertile che si è respirata. $\mathrm{Ne}$ è emersa una comunità idrogeologica italiana non solo numerosa, ma anche desiderosa di confrontarsi al suo interno e verso l'esterno, con le sfide e le problematiche che la società, l'ambiente e il periodo di crisi ci pongono di fronte. E' però necessario l'impegno di tutti affinchè queste potenzialità, certamente non inferiori a quelle degli altri paesi leader in campo idrogeologico, non vadano disperse ed anzi convergano il più possibile verso obiettivi comuni. In attesa della prossima edizione annunciata per Viterbo 2014, non ci resta che seguire tutti insieme i futuri "Flowpath"!

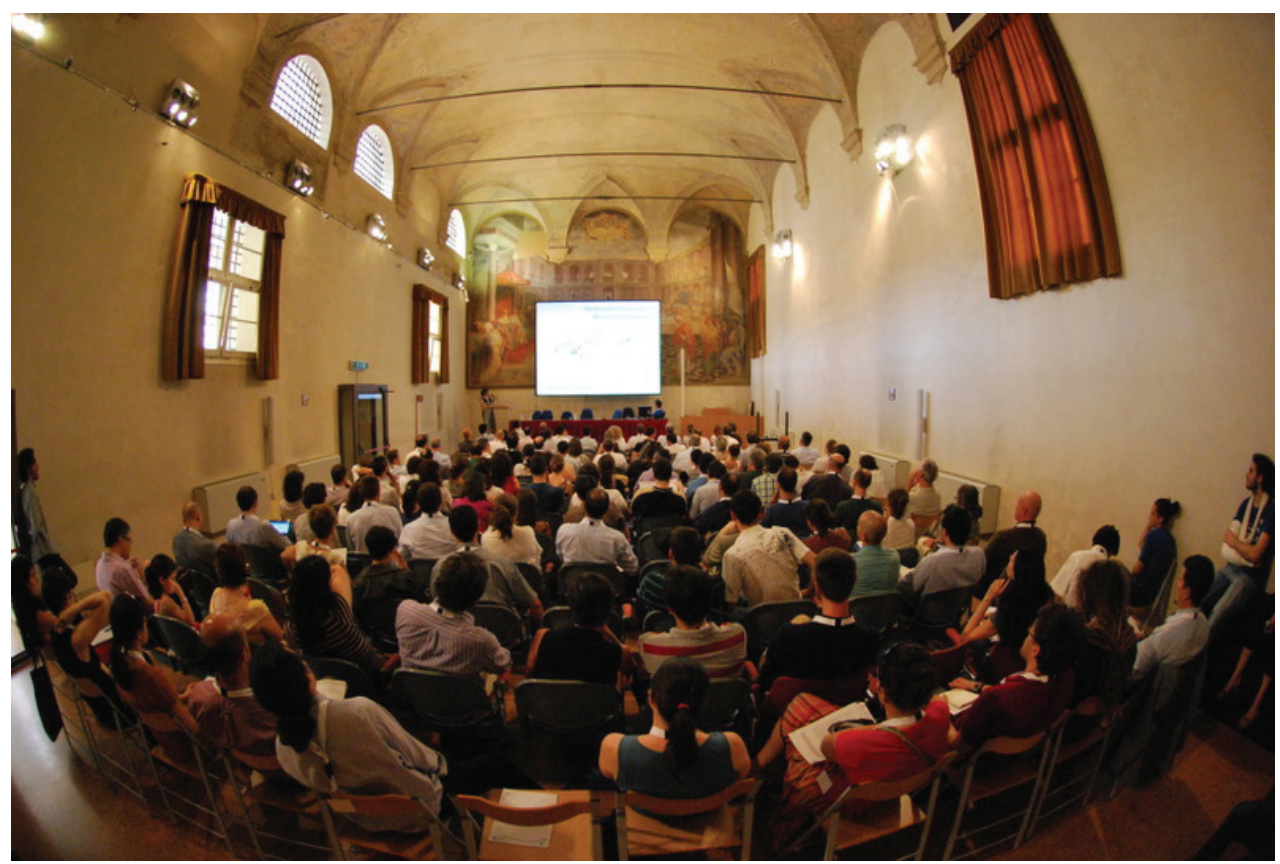

Fig. 2 - Platea durante le presentazioni 